\title{
CONTROVERSIAS EN TORNO AL SOFTWARE LIBRE: PRÁCTICAS Y DISCURSOS EN LA DOCENCIA UNIVERSITARIA VALENCIANA
}

\author{
(CONTROVERSIES ABOUT THE FREE SOFTWARE: PRACTICES AND SPEECHES IN THE \\ UNIVERSITY VALENCIAN TEACHING)
}

\author{
Ángel San Martín Alonso \\ José Peirats Chacón \\ Cristina Sales Arasa \\ Universitat de Valéncia (España)
}

\section{RESUMEN}

La progresiva implantación del software libre en los ámbitos universitarios, se acompaña de un discurso construido sobre las controversias que rodean a esta tecnología. Para el análisis de este problema utilizamos el modelo de "espacios controversiales" propuesto por Nudler, definiendo uno de estos espacios en torno a la aplicación de las tecnologías informáticas en las enseñanzas universitarias. El supuesto es que el debate sobre el copyleft está alcanzando también al discurso pedagógico que acompaña a las aplicaciones de estas tecnologías. Este modelo es el que hemos tratado de aplicar al estudio de cómo se formulan tales controversias en la difusión del software libre en la docencia de las universidades públicas valencianas. En esta primera aproximación quizá el resultado más llamativo es que las controversias no alcanzan a la esfera pública, se restringe al ámbito de los técnicos, mientras la institución asume costes adicionales para su implantación.

Palabras clave: software libre, controversias, pedagogía, enseñanza superior, tecnologías informáticas.

\begin{abstract}
The progressive introduction of the free software in the university areas, it's accompanied of a speech constructed on the controversies around this technology. For the analysis of this problem we use the model of "controversial spaces" proposed by Nudler, defining one of these spaces about the application of the computer technologies in the university educations. The supposition is that the debate about the copyleft is reaching also to the pedagogic speech that accompanies on the applications of these technologies. This is the model we have tried to apply to the study of how such controversies are
\end{abstract}


formulated in the diffusion of the free software in the teaching of the public Valencian universities. In this first approximation probably the most showy result is that the controversies do not reach to public sphere, is restricted to the area of the technical personnel, while the institution assumes aftercosts for it introduction.

Key words: free software, controversies, pedagogy, higher education, computer technologies.

\section{EL SOFTWARE LIBRE COMO CONTROVERSIA PEDAGÓGICA}

La necesidad de utilizar en la enseñanza superior los recursos que proporcionan las tecnologías de la información, es algo universalmente reconocido y considerado en sí mismo como valioso. Coyuntura que reclama una pedagogía capaz de aportar elementos conceptuales para encajar las nuevas formas de enseñar y aprender mediante las TIC. En este contexto, nos posicionamos desde el principio, entendemos por pedagogía el conjunto de conocimientos que presta atención tanto a la producción como a la reproducción del saber. Dado que además nuestro interés se centra en el ámbito institucional de la universidad, la pedagogía remite inexorablemente al cómo se enseña, qué se enseña y, asunto fundamental, al cómo se aprende (Gore, 1996, p. 22 y ss.). Estas tres dimensiones del "problema pedagógico" adquieren nuevos caracteres cuando intervienen las tecnologías de la información. Ahora bien, articular metodológicamente todos esos elementos no sería tan problemático como cuando se adquiere conciencia de la vertiginosa y radical evolución de las TIC. Su dinámica innovadora dificulta establecer esquemas estables desde los que ordenar la incorporación de las tecnologías a los procesos de enseñanza y aprendizaje, sea en la universidad o en cualquier otro nivel del sistema escolar.

Más allá de la aceptación acrítica del valor “pedagógico” de las TIC, consideramos que su presencia en las instituciones educativas y también en la universitaria, constituye un núcleo problemático que trasciende lo meramente instrumental y los planteamientos más o menos positivistas que hoy dominan la mirada científica sobre la enseñanza y el aprendizaje. En este contexto el discurso pedagógico se retrae y supedita al que dictan los agentes más activos del entorno tecnológico. De este modo se está llegando a un punto en el que la pedagogía la formulan los diseñadores gráficos y los ingenieros de sistemas informáticos, quienes sitúan el objeto pedagógico en el terreno que les es más propicio. Sin embargo, los resultados, salvo los económicos, suscitan demasiados interrogantes como para abandonar la empresa de repensar la pedagogía que, con la presencia de las tecnologías informáticas, ha de aportar luces sobre cada unas de las tres dimensiones mencionadas más arriba. 
El movimiento social que acompaña al software libre (SL en lo sucesivo), dentro y fuera de los ámbitos académicos, invita a pensar que su área de incidencia trasciende lo tecnológico para alcanzar el plano pedagógico. Somos conscientes que aún es pronto para observar ese fenómeno, por eso nos proponemos analizar cómo se produce este salto cualitativo a través de las controversias movilizadas en los discursos y las prácticas universitarias con las tecnologías informáticas. Recordemos, antes de nada, que las tecnologías en la sociedad actual despiertan no pocas controversias y algunas de calado estructural, tal como argumenta Martín Gordillo (2006). La opción por un software libre o propietario no es en absoluto coyuntural, sino estratégica para la institución. Y lo es porque no sólo se han de considerar factores de naturaleza económica, sino que ha de atender también a la interconectividad de los sistemas, a los de índole social y política que acompañan a esta tecnología. Pero en la Universidad se conjugan, como mínimo, cuatro funciones (investigación, docencia, gestión y extensión cultural), en las que el apoyo de las TIC es consustancial. De manera que la opción por uno u otro de los sistemas operativos se convierte en algo fundamental para su competitividad. En cualquier caso, aquí nos ocuparemos prioritariamente de las controversias que rodean a la opción tecnológica en el ámbito de la docencia por las implicaciones pedagógicas que hipotéticamente pensamos va a tener.

\section{UN MODELO PARA COMPRENDER EL PROBLEMA}

La innovación de las tecnologías en la enseñanza institucionalizada, aparte de la euforia difundida por el discurso dominante, plantea no pocos problemas y controversias. Alentados por la creciente expansión del SL, aparecen nuevos elementos para el debate en un campo ya bastante problemático. Pero la emergencia de estos nuevos recursos tecnológicos no surge por generación espontánea, sino vinculados al intenso y complejo proceso de innovación del sistema técnico. Innovación que ahora, como el resto de innovaciones, se pretende generalizar a través de los sistemas escolares. Ahora bien, la presencia del software no propietario en las aulas representa, a nuestro entender, un caso específico que merece la pena tratar con atención. Por esta razón echamos mano de un modelo que nos ayude a comprender y conceptualizar lo que puede representar este tipo de software en la enseñanza, concretamente en los ámbitos universitarios valencianos.

El modelo aludido es el de los campos o espacios controversiales, propuesto por Nudler (2004) como intento de superar las debilidades del modelo clásico de Kuhn en la explicación del desarrollo de la ciencia y la tecnología. Por lo 
pronto, del modelo alternativo destacaríamos de entrada dos ideas importantes: las controversias, en ningún campo científico, van aisladas sino que están estrechamente relacionadas entre sí y, por otra parte, esta relación hace que en el desarrollo científico y tecnológico, también en el campo que nos ocupa, no se produzcan verdaderos saltos en el vacío, cambios radicales de paradigmas según el planteamiento kuhniano. La aparición de las controversias en un campo y sus relaciones van induciendo cambios en el campo sin desprenderse totalmente de los supuestos y hallazgos precedentes. Hay un encadenamiento entre las controversias que, pese a no ser continuo ni constante, hace progresar al conocimiento y la tecnología disponibles. Este sería el caso del SL respecto al propietario, por cuanto aquél representa un avance técnico y, sobre todo, en la socialización de la tecnología respecto al protegido por el copyright.

Según el trabajo ya citado de Nudler (2004, p. 12), el espacio controversial suele tener "una estructura interna bastante intrincada", dada la relación establecida entre las controversias que se dan cita en ese espacio. Ahora bien, pese a la complejidad de la estructura, hay siempre dos ámbitos diferenciados en esos espacios. Por un lado, estaría el ámbito "de los problemas que están en el foco de las diversas controversias que integran el espacio considerado", mientras que el otro ámbito del espacio estaría ocupado por "los compromisos metafísicos, metodológicos, teóricos que los actores comparten o aceptan, ya sea de manera explícita o implícita, y que, por tanto, no son objeto de controversia", corpus que denomina common ground y cuya variación es mucho más lenta en el tiempo que lo situado en el foco. En el esquema de la Figura 1 tratamos de sintetizar este modelo aplicado al problema que nos ocupa, así como su repercusión teórica y práctica en la enseñanza superior.

De acuerdo con el esquema, la controversia que gira en torno a si el software debe ser libre o propietario, de inmediato se relaciona con otras, no menos relevantes, cuyo nexo remite a un campo más amplio que denominamos como "Tecnologías informáticas en la enseñanza ${ }^{1}$. En torno a este enunciado problemático se perfila el espacio controversial configurado por una serie de disputas o focos, que comparten una serie de supuestos teóricos y metodológicos sobre la pertinencia pedagógica de las TIC, como es el caso de Carnoy, 2004; De Pablos y Villaciervos, 2005; Area Moreira, 2004, 2000. Pero estas asunciones compartidas se mantienen en diálogo con una serie de focos problemáticos, a los que no se les presta la misma atención ni ésta deriva de los mismos campos científicos. En el esquema señalamos cinco focos de controversias aunque podrían identificarse algunos más, si bien los apuntados son suficientes para analizar el ámbito universitario que aquí nos interesa. 
Siguiendo el modelo de Nudler (2004, p. 13), uno de los focos, en un momento determinado y por razones que ahora no podemos detenernos a revisar, entra en juego como un nuevo participante "que desafía a los jugadores tradicionales poniendo sobre la mesa y criticando todos o buena parte de los supuestos implícitos sobre los cuales reposaba hasta ese momento el debate". La pujanza de esta presencia remueve el terreno común del espacio controversial haciéndolo cambiar, total o parcialmente, tanto los supuestos como los focos de debate. A este cambio es a lo que el filósofo citado denomina refocalización.

En nuestro caso el "nuevo jugador", sin duda, viene representado por el llamado SL y cuya pujanza invita a revisar los supuestos sobre los que se sustenta el espacio controversial que nos ocupa. Es más, a modo de hipótesis mantenemos que esta reestructuración implicará la revisión conceptual y teórica del marco pedagógico que da cobertura a los discursos y las prácticas sobre las TIC en la enseñanza. En este sentido no dudamos en reconocer el potencial que entraña la refocalización alentada por el software no propietario para el espacio controversial que nos ocupa. De ahí la importancia de observar cómo este espacio se objetiva en un ámbito de referencia para la sociedad y los demás niveles escolares, como es el de la enseñanza universitaria. Pero antes de entrar en la cuestión de fondo, haremos unas breves consideraciones sobre la presencia de las TIC en la Universidad y concretaremos a qué nos estamos refiriendo cuando hablamos de SL.

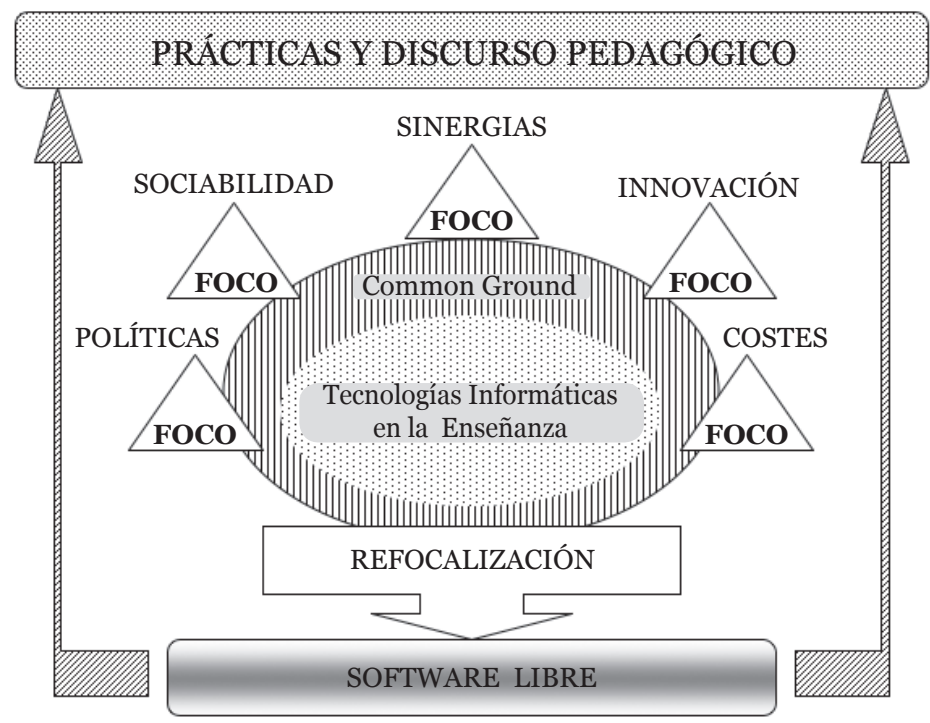

Figura 1. Espacio controversial sobre las TIC en la enseñanza 


\section{EL CONTEXTO UNIVERSITARIO ANTE LAS TIC}

La institución universitaria se encuentra inmersa en la denominada Sociedad de la Información, y ésta le está exigiendo una serie de cambios, le demanda que se adapte a las nuevas características que están adquiriendo todos los ámbitos de dicha sociedad (cultural, económico, educativo,...). En la exposición de motivos de la Ley Orgánica ${ }^{2}$ por la que se modifica la LOU (2001), se alude a la necesidad de que las universidades respondan con flexibilidad y rapidez a los cambios en las necesidades de una sociedad dinámica y globalizada y obviamente sabemos que uno de los cambios gira en torno al protagonismo que han adquirido las TIC en muchas de las actividades que se desarrollan en la sociedad. En la misma línea, el documento marco titulado $L a$ integración del sistema universitario español en el espacio europeo de enseñanza superior (MECD, 2003, p. 3) $)^{3}$, se insta a una nueva ordenación de la actividad universitaria, puesto que la "sociedad del conocimiento requiere innovaciones y cambios en las formas tradicionales de formación, producción, comunicación de la información y en el acceso a servicios públicos y privados". De este modo, la aplicación del Sistema Europeo de créditos de transferencia y acumulación (ECTS) comportará muchos cambios en la enseñanza universitaria, y en el perfil de sus docentes; González (2005, p. 44) apunta que una de las competencias que se ajustarán a dicho perfil será la capacidad de integrar las TIC en los procesos formativos.

Se apuesta pues en diversos documentos por la introducción de las TIC en la institución universitaria y concretamente se asume que pueden cambiar el proceso de enseñanza-aprendizaje, la docencia universitaria va a verse transformada al integrar estos artefactos. Ahora bien, creemos al igual que Marrero (2004, p. 41) que "la cuestión clave ahora es cómo va a afrontar la Universidad la adaptación a los nuevos cambios", y puesto que toda adaptación parte de una situación inicial, nos preguntamos ¿̇cuál es la realidad? ¿cuál es el nivel de penetración de las TIC en la docencia universitaria? No es objeto de este trabajo responder a estas preguntas, puesto que ello excedería nuestras pretensiones. Sí que podemos decir que cada vez son más los docentes que incorporan TICs a su enseñanza, a pesar de datos no muy halagüeños como los recogidos en el informe España 2006 (2007, p. 294) 4: "el uso de las TIC por parte de la comunidad científica universitaria ha sufrido un cierto estancamiento". No es más esperanzador el panorama descrito por Cabero (2002, p. 300), quien tras un estudio sobre dotación y uso de TICs en las Universidades de Sevilla, Santiago, Rovira i Virgili, País Vasco, Murcia e Islas Baleares, afirma que los profesores las utilizan para "tareas usuales y en cierta medida tradicionales, como por ejemplo para motivar a los estudiantes, acceder a más información o presentársela a los estudiantes". Por tanto, de todas las funcionalidades que ofrecen los "campus 
virtuales", que bien describe Marqués (2000, p. 5), el docente se decanta por unas pocas herramientas: el correo electrónico, alojar y distribuir materiales docentes, etc. Y estamos hablando del profesorado que utiliza estas plataformas, que según el informe de la Conferencia de Rectores de Universidades Españolas Las TIC en el sistema universitario español: un análisis estratégico (2006), alcanza únicamente un $43 \%$, a pesar de que un $96 \%$ de los docentes tiene acceso a la Red.

No obstante, a nivel institucional sí que se está dando un gran impulso al uso de las TIC y esto se traduce sobre todo en un aumento de la oferta de formación online, de manera que se apuesta fuertemente por el modelo de enseñanza-aprendizaje mixto, también denominado blended learning. El informe citado nos muestra que un $87 \%$ de las universidades españolas posee un plan institucional de docencia virtual, aunque también nos hace saber que sólo el $37 \%$ de las asignaturas impartidas apoyan las clases presenciales mediante la utilización de alguna plataforma software de uso educativo. En esa línea, Martínez constataba en 2002 que de las 50 universidades públicas españolas, 17 tenían alguna asignatura semipresencial o virtual. Y más cercano en el tiempo, Valverde et al. (2004) concluye que la oferta académica de dichas universidades a través de las redes telemáticas para el curso 2003-2004 triplica la del curso 2001-2002 y es mayor en el área de Ciencias Sociales que en otras. Los autores apuntan que la tendencia hacia este modelo se debe, en primer lugar, a un intento de mejorar el proceso de enseñanza-aprendizaje y en segundo lugar, a la reducción de costes que supone para las universidades (Valverde et al., 2004, p. 115). Ante lo cual nos preguntamos: ¿está probada dicha mejora?; en cualquier caso, ¿cuál de las dos razones tiene un mayor peso a la hora de impulsar este modelo de enseñanza?

Y en el marco de tal interés por el blended learning y por la utilización de las herramientas telemáticas en la docencia universitaria, está adquiriendo un gran protagonismo el conocido como software libre. Pero ¿̇a qué nos referimos con esta denominación? ¿Qué aporta de diferente o novedoso frente al software propietario? ¿Cómo está penetrando en el ámbito universitario? Seguidamente tratamos de ofrecer algunas respuestas a estas cuestiones.

\section{UN "NUEVO JUGADOR" EN EL ESPACIO CONTROVERSIAL: EL SOFTWARE LIBRE}

En el espacio configurado en torno a las virtualidades de las tecnologías informáticas en la enseñanza (Fig. 1), irrumpe con fuerza, por diferentes razones, un actor que está refocalizando todo el espacio controversial. En efecto, nos 
referimos al software libre y su presencia en los ámbitos educativos, especialmente en los universitarios. El SL comprende aplicaciones informáticas alternativas a los programas denominados propietarios, como por ejemplo Windows. La Free Software Fundation (FSF) lo define como aquel software que permite las siguientes libertades a los usuarios ${ }^{12}$ :

- Usar el SL como ellos quieran, para lo que quieran, en los ordenadores que ellos quieran, en cualquier situación apropiada técnicamente.

- Tener el SL a su disposición para ajustarse a sus necesidades.

- Redistribuir el SL para otros usuarios, los cuales podrían usarlo de acuerdo a sus propias necesidades.

- Los usuarios de un software determinado deben tener acceso a su código fuente.

Su principal característica es pues que se basa en estándares abiertos, frente al software propietario que lo hace sobre estándares cerrados, de modo que cualquiera puede utilizar y desarrollar una determinada aplicación de SL porque tiene acceso al código fuente. Ahora bien, el fenómeno del SL no es nuevo; existe una larga trayectoria histórica, desde que un grupo de investigación de la Universidad de California mejorara el Sistema Unix creado en 1974, dando lugar al "BSD Unix". En 1984 Stallman lanza el GNU Project, que pretendía ser un sistema operativo y por esos años se crea la Free Software Fundation. Y siguiendo estos pasos, en 1991 Linus Torvals aporta a GNU Project un kernel o núcleo de sistema operativo similar a Unix, con lo cual nace el sistema operativo GNU/Linux.

Son hitos fundamentales para entender lo que ya se puede denominar un movimiento social, descrito por Himanen (2002) a través de la "ética hacker" que lo caracteriza. Colectivos de personas participan de manera desinteresada y colaborativa en el desarrollo y mejora de los códigos fuentes, a crear aplicaciones que respondan a las necesidades de los usuarios. "Se trata de un espíritu general: los hackers programan porque para ellos tiene un interés intrínseco. Los problemas relacionados con la programación despiertan una curiosidad genuina en el hacker y suscitan su deseo de continuar aprendiendo" (Himanen, 2002, p. 23). Ahora bien, no todo es altruismo; hay también empresas de la talla de IBM o HP que se han interesado por el SL en tanto pretenden disputar cuota de mercado a Microsoft y sobre todo al basar su negocio como apunta Fernández (2005, p. 67), "en la distribución de programas libres, en especial el Sistema Operativo Linux, obteniendo ingresos no por la entrega del sistema operativo en sí sino por servicios tales como asistencia técnica, consultoría o formación". 
Este movimiento social está introduciéndose a nivel institucional y no nos referimos únicamente al contexto español. En el marco europeo, el Plan de Acción eEurope promueve el fomento de la utilización de programas de fuentes abiertas y son muchos los países que llevan a cabo iniciativas en ese sentido ${ }^{13}$. Una de las razones que se apuntan ${ }^{14}$ para ello radica en la competitividad que puede ganar Europa frente a Estados Unidos, en la independencia tecnológica, como se aduce también en el informe Free Software/Open Source: Information Society Opportunities for Europe? (2000) elaborado por un grupo de trabajo a propuesta de la Comisión Europea. Y estas directrices europeas en nuestro país se traducen en iniciativas propias y diversas en cada Comunidad Autónoma, algunas centradas en el ámbito educativo, como el Lliurex en la Comunidad Valenciana, y otras generalizadas a la educación y a toda la Administración, como es el caso del Linex en Extremadura.

Ahora bien, volviendo al tema que nos ocupa, ¿cuál es la penetración real del SL en el ámbito universitario? Bien, es cierto que las Universidades españolas están teniendo un gran peso en la difusión del SL. Hay muchos proyectos destacables, por ejemplo la Universidad de las Palmas de Gran Canaria crea una Oficina del Software Libre con la idea ${ }^{15}$ de hacer de la universidad "un ejemplo de reutilización de los propios recursos, de un mejor aprovechamiento de los fondos públicos y de una apuesta por la innovación tecnológica y la pluralidad”. En la misma línea y en el marco de la Universidad Autónoma de Madrid, la Asociación para el Fomento del Software Libre pretende objetivos similares, y más allá de su propio contexto intenta, con el Proyecto Anillo Web Linux/SL Universitario "crear las comunicaciones, relaciones y colaboración entre las asociaciones, clubes y grupos universitarios relacionados con el SL" ${ }^{\prime 6}$. Una de las Universidades que colabora en este proyecto es la Jaume I de Castellón. Precisamente esta Universidad, al igual que la Universidad de Oviedo, da un paso más y plasma en sus Estatutos el compromiso de velar por la difusión del SL: "La UJI fomentará el uso de formatos informáticos abiertos en la comunicación interna y externa, promoverá el desarrollo y el uso de SL y favorecerá la libre difusión del conocimiento creado por la comunidad universitaria" (p. 138)

Cada vez son más las iniciativas y los proyectos alrededor de esta nueva tecnología, aunque como se afirma en el informe eEspaña 2005 "no existe información detallada sobre el grado de penetración y usos del software libre en las universidades españolas" (p. 333). La verdad es que poco a poco van apareciendo nuevos datos al respecto, más referidos a la disposición de dicho software que a su utilización en la docencia. Según el informe Las TIC en el sistema universitario español; un análisis estratégico (2006, p. 142) "el 30\% de los productos utilizados 
en las universidades son, por término medio, de software libre, produciéndose una importante expansión en uso con respecto a 2004. El porcentaje de ordenadores que tiene instalado un sistema operativo de libre distribución alcanza el 10\%” Figuerola y otros (2007, p. 93) analizan los sitios web de 72 universidades españolas y concluyen que "el SL tiene un grado notable de implantación a nivel de servidores o hosts" aunque también destacan que los formatos de ficheros más utilizados siguen siendo los basados en estándares cerrados: "el uso de la suite ofimática propietaria de Microsoft sigue siendo mayoritaria, y esto repercute en los formatos de los ficheros que se cuelguen en la red" (Figuerola y otros, 2007, p. 90). Es interesante saber que muchas veces, detrás de estos proyectos se encuentra la iniciativa privada; la Universidad Politécnica de Madrid crea en 2001 el Centro de Referencia Linux junto con la empresa IBM, y en la Universidad Rey Juan Carlos se firma un acuerdo con la empresa Lambdaux para poner en marcha el Plan Integral de implantación de Software Libre.

Es pues un hecho que el SL se va haciendo presente en la Universidad, en diversas formas y proyectos, impulsado a nivel institucional y también por grupos de profesores interesados en la difusión y uso de esta nueva herramienta. Y en el contexto de la Comunidad Valenciana, y aunque ya hemos aludido a alguna de sus universidades, cabe destacar que no hace mucho y como consecuencia de la introducción del Lliurex ${ }^{18}$ en las escuelas y los institutos valencianos fuimos sorprendidos por la firma de un convenio entre la Conselleria de Educación autónoma y algunas de las universidades valencianas, concretamente con la Universitat d'Alacant, la Universitat de València y la Universitat Jaume I de Castelló. Fruto de ese primer convenio algunos docentes de las citadas universidades han elaborado una serie de cursos de autoformación en línea; en primer lugar para el adiestramiento de los alumnos de Magisterio en SL, en segundo lugar para la distribución por los colegios e institutos de la comunidad autónoma, y por último para la libre disposición de los docentes y el público en general ${ }^{19}$. Y no acaba aquí la cooperación, en el futuro se prevé nuevos convenios para elaborar, entre otros, nuevos cursos sobre Lliurex avanzado, la creación de materiales didácticos multimedia y la generación de sitios web con Lliurex.

\section{FOCOS DE CONTROVERSIA SOBRE EL SL EN LA ENSEÑANZA UNIVERSITARIA}

Una de las consecuencias de la refocalización es que moviliza epistemológica y discursivamente los focos que constituyen el espacio controversial. Por ello nos proponemos ahora revisar cada uno de los focos identificados en la Figura 1, a fin de 
analizar cómo se redefinen en los documentos y proyectos que algunas universidades valencianas tienen sobre la implantación del SL. Pretendemos asimismo identificar los nexos y relaciones establecidos entre ellos, para que nos permitan entrever la estructura sobre la que se constituye el espacio controversial que nos ocupa.

\section{Sinergias entre opuestos}

En la sociedad digitalizada dominante no existe la unanimidad entre los usuarios en cuanto a la selección y la utilización de las distintas aplicaciones en SL, tanto si nos referimos a los sistemas operativos, las aplicaciones ofimáticas, las referidas al ocio o aquellas que persiguen la información y la comunicación, y especialmente si nos fijamos en el origen y la naturaleza de las mismas. En ese sentido y como prueba de ello encontramos, en la selección de los programas, la bipolarización cada vez más tangible entre los partidarios del código abierto y los partidarios del código propietario. Entre estos dos grupos se han ido poco a poco incrementando las discusiones entre sus partidarios, hasta el momento plasmadas en algunas voces contrapuestas reflejadas en los diferentes medios de comunicación.

Sin embargo, en cuanto a la composición e importancia de estos sectores no podemos afirmar que sean comparables y la preponderancia del código propietario hasta el momento no se ve alterada más que en algún aspecto marginal. Estadísticamente, es cierto que el sistema operativo Windows se encuentra en más del 90\% de los ordenadores domésticos de todo el mundo, y sólo el sistema operativo basado en SL, Linux, le hace sombra en el mercado de los servidores empresariales con casi el 30\% del mercado ${ }^{5}$. El posible usuario de este segundo sistema, y más si tiene carácter doméstico, a la hora de comprar equipos con el sistema operativo instalado encuentra muchas dificultades en los establecimientos del ramo; la mayoría lo ofrecen con el nuevo Vista instalado y se niegan a vender los ordenadores sin sistema operativo o con el Linux.

Con todo podemos anotar algunos movimientos a favor del sistema libre en empresas productoras de ordenadores personales, como es el caso de Dell que ofrecerá, bajo pedido, la posibilidad de instalar Linux en lugar de Windows. Otra muestra de esta tendencia, en este caso el de la telefonía móvil, la encontramos en que varias compañías importantes ${ }^{6}$ se han unido con el objetivo de desarrollar conjuntamente software para móviles basados en código abierto, concretamente la estandarización y la adopción de Linux sobre los teléfonos móviles. Y también, la aparición reciente de las aplicaciones online gratuitas, como las de Yahoo o de Google, para la edición de documentos, hojas de cálculo, retoque de fotografías, etc. que 
dejan en segundo plano a las que adquirimos para nuestro ordenador. O, en cuanto a una de las aplicaciones de uso diario, el espectacular aumento en la utilización del navegador de código abierto Firefox de Mozilla, y en contra de Internet Explorer instalado en todos los ordenadores suministrados con Windows.

A pesar de esta todavía más que evidente desproporción hay un crecimiento que algunos opinan que es imparable, y esgrimen como argumentos que el sistema de Linus Torvals ofrece razones de ahorro de costes, más su seguridad y estabilidad como pruebas suficientes que demuestran la positiva evolución de una comunidad que ha basado su funcionamiento en la "apertura de código"; es decir, en la aportación y la colaboración entre todos para alcanzar la revisión y discusión permanente. Y no sólo en cuanto al sistema operativo, la suite de ofimática o el navegador a utilizar; el desarrollo alcanza ya todos los ámbitos de desarrollo de software, y como botón de muestra podemos nombrar uno de los proyectos de la Fundación Wikimedia, la enciclopedia libre Wikipedia ${ }^{7}$, libro colectivo que cuenta con un sistema que garantiza el acceso a sus contenidos, la actualización del conocimiento y la democracia en su proceso de edición.

Siguiendo a Angás (2007) ${ }^{8}$, los partidarios de los estándares abiertos aceptan la convivencia pasada, presente e incluso también futura con el código privado pero subrayan a favor de sus ideas que la tecnología, tal como lo hicieron con anterioridad otros sectores, se está estandarizando para ser más productiva y para alcanzar la utilización racional de los recursos. En este sentido muestran como ventajas del software, de autor pero sin dueño, el que facilita que los ordenadores trabajen entre sí, ayuda a reducir la posibilidad de fallos del sistema y gestiona la complejidad de las interconexiones electrónicas mundiales.

Esta controversia también alcanza al ámbito universitario donde coexisten, al alcance y para el trabajo de profesores y también de alumnos, programas con licencias de campus y plataformas para la ayuda en la docencia que se pueden enmarcar tanto en una opción como en la otra. Aunque esta variedad de oferta nos tememos oculta otros intereses tal vez no demasiado acordes con el fin y los objetivos de la institución universitaria.

Por tanto, lo que ocurre es que, de momento y por mucho que desde la Universidad se esté difundiendo el SL, los alumnos e incluso el profesorado sigue utilizando en sus domicilios muchas aplicaciones de software propietario. En esta paradoja encontramos el problema de la interoperabilidad entre herramientas, ya que al usuario de un sistema operativo lo que le interesa realmente es que pueda abrir 
correctamente un archivo con sus programas, independientemente del ordenador desde el que se lo envían. Además, existen aplicaciones muy específicas, como por ejemplo Autocad o SPSS, cuyo potencial operativo, de momento, no tiene parangón en SL, con lo cual todavía se complica más la elección.

\section{Los costes del SL}

Hay que decir que una de las ventajas más proclamadas del SL es el hecho de que se reducen costes al consumidor en tanto no existen licencias del estilo de las del software propietario. Sin embargo, el hecho de no pagar licencias no significa que el negocio del SL no genere beneficios. Como hemos señalado anteriormente, y también como apuntan Romeo y García (2003, p. 155) "las empresas de SL ganan dinero por los servicios relacionados con la consultoría, desarrollo de software, formación, soporte y gestión de las aplicaciones". Por tanto, es un tanto aventurado argumentar que el ahorro económico en licencias revertirá en una mayor dotación para ámbitos como la formación del profesorado.

En cualquier caso, ¿̇se está dando la formación necesaria al docente universitario de la Comunidad Valenciana para que se utilice el SL? Revisando la oferta formativa de las principales universidades, sí que hay que destacar que todas incluyen algún curso sobre el uso de SL en la docencia; así, en la Universidad Jaume I y dentro del apartado de formación denominado "Herramientas tecnológicas para el apoyo de la docencia a la UJI", encontramos un curso de 8 horas de duración, "Iniciación al aula virtual de la UJI". En el ICE de la Universidad de Alicante aparecen cursos específicos referidos al SL, como "Herramientas de evaluación en campus virtual", "Gestión de currículum de investigación a través del Campus virtual" o "Sesiones docentes en campus virtual". También se celebran en dicha Universidad las Jornadas de Innovación Tecnológica-Educativa y las Jornadas Copla (Jornades pel coneixement obert i programari lliure a la universitat d'Alacant). Actuaciones similares encontramos en la Universidad de Valencia, como por ejemplo, las Jornadas sobre Aula Virtual.

\section{Fomento de la sociabilidad entre usuarios y desarrolladores}

Romeo y García (2003, p. 149 y ss.) señalan que el SL "promueve la libertad y la cooperación y por ello la comunidad educativa, aprovechándose de toda una infraestructura tecnológica creada en base a estos dos valores, transmite el ejemplo de la cooperación como base de las relaciones sociales". Obviamente no vamos a ser nosotros los que renunciemos a tan noble propósito, si bien cabe decir que el ámbito 
universitario siempre ha defendido estos valores, al margen de las tecnologías utilizadas. Como afirma Marrero (2004, p. 45) "la colegialidad, la colaboración, la reflexión y la internacionalidad conforman, también en la Universidad, los nutrientes de su cultura organizativa”. Además, ahondando en el tema de la tan proclamada cooperación entre los integrantes de la comunidad del SL, parece que muchas veces no lo sea tanto: a partir de un proyecto inicial y debido a intereses distintos o discrepancias entre las personas que lo desarrollan, se crean subproyectos o diversas aplicaciones de un mismo proyecto, fenómeno ya conocido como bifurcaciones o fork. Algunos ejemplos son la escisión entre la Enciclopedia Libre y la Wikipedia en español, o el proyecto GoneME, fork del escritorio GNOME, que intenta realizar un ex-miembro de la Fundación GNOME aduciendo entre otras razones ${ }^{9}$ la poca atención que los desarrolladores de dicho escritorio dan a los usuarios.

Otra delas ventajas queaquellos autores apuntan es queelSL “eslabasetecnológica para la innovación en la comunidad educativa, de modo que el colectivo de educadores podrá diseñar las funcionalidades a implantar desde su propio conocimiento de la educación". Ahora bien, nos cuestionamos si el profesorado universitario en general aprovechará esta posibilidad de diseñar sus propias aplicaciones. Quizá esto sea una ventaja para las empresas, las administraciones públicas o los profesionales de la Informática, pero habrá que ver si el docente universitario en particular se involucra en semejante tarea. Nos preguntamos, al igual que Fernández (2005, p. 66) “¿a quién que no sea un especialista en la programación informática puede interesarle el segundo trío de libertades que cita la FSF (estudiar, cambiar y mejorar un programa informático)?”.

\section{Política universitaria alrededor del SL}

La colaboración ente la Administración y las universidades, que se plasma en nuestro caso en el convenio al que anteriormente aludíamos entre Conselleria de Educación y algunas universidades valencianas, cuanto menos y a priori hay que celebrarla ya que no son sobradas las experiencias en ese sentido. Sin embargo, tal vez supongan el alineamiento de la universidad con planteamientos partidistas tecnológicos que puedan contradecir algunas de las prácticas usuales en las mismas, o el posicionamiento con determinadas aplicaciones que, aunque de libre distribución, puedan suponer nuevos espacios de poder y para el control del trabajo con los medios tecnológicos. En este importante tema, y en cuanto a la filosofía que subyace detrás del código libre, no nos olvidemos que la utilización de determinados programas implica a veces la pertenencia a comunidades que forman auténticas redes mundiales, con 
el consiguiente esfuerzo para la contribución al desarrollo de los mismos; o incluso caer bajo la tutela de empresas multinacionales que las distribuyen.

Al revisar los repositorios tecnológicos de las tres universidades citadas y pasamos al campo de los entornos virtuales de enseñanza observamos, en principio, que si bien utilizan las tres la misma plataforma en cuanto a los cursos suscritos en el convenio con Conselleria, en cambio no coincide con la herramienta seleccionada para apoyo a la docencia universitaria en alguna de estas instituciones. ¿Por qué han elegido una determinada plataforma y no otra? ¿Qué razones les mueve para la utilización masiva de unas herramientas que aún siendo de código libre también están sujetas a condicionamientos e intereses propios? En definitiva, ċhacia dónde va la universidad valenciana en el proceso de introducción del SL? Estas contradicciones merecen también nuestro interés y, en primer lugar, parece pertinente describirlas aunque sea someramente, y analizar los motivos o razones que les han movido a decantarse por cada una de ellas.

En primer lugar hacemos referencia a la Universitat d'Alacant, que a través del denominado Campus Virtual ofrece un servicio de complemento a la docencia y a la gestión académica y administrativa, dirigido tanto al profesorado como al alumnado y al personal de administración. Es una plataforma cuya principal característica reside en el hecho de que ha sido desarrollada con recursos y personal propio; en la que, y dejando de lado las opciones de gestión disponibles para todo el personal de la universidad, el profesorado puede obtener listas de clase, visualizar las fichas de los alumnos, realizar tareas de tutoría, proponer y moderar debates, y alojar todo tipo de materiales para sus alumnos: documentos, bibliografía... Se complementa, en el afán de impulsar las iniciativas para dar a fomentar el SL en la universidad, con el proyecto COPLA (Coneixement Obert i Programari Lliure a la Universitat d'Alacant) que pretende mediante una serie de fases progresivas la difusión del conocimiento abierto, en general, y del SL, en particular. Objetivos que se concretan en iniciativas tales como el desarrollo de campañas informativas, organización de eventos, convenios de colaboración, incremento de la formación y del uso de formatos libres...

Esta apuesta por el SL encuentra su razón de ser en una serie de ventajas que esta universidad concreta, en función de su especificidad lingüística, en que este software al tener el código accesible puede ser fácilmente traducido al valenciano. También en la calidad de los programas, al ser muchas las personas que tienen acceso al código se subsanan los problemas con mayor rapidez. Además de mayor privacidad, seguridad y garantía de continuidad al ser los propios técnicos de la universidad los que mantienen el programa. Otra de las ventajas la centran en el ahorro de costos, en 
cuanto a la compra de licencias, y relacionado con el tema la distribución libre entre los alumnos. Por último, encuentran en este campo ventajas para la cooperación con otras universidades para compartir, difundir y fomentar estas ideas.

En la Universitat de València la aplicación de apoyo a la docencia se denomina Aula Virtual. Esta plataforma tecnológica de gestión del aprendizaje se basa en .LRN, un sistema de SL desarrollado por una comunidad mundial en la que se integran distintas universidade ${ }^{10}$ y que además utiliza una base de datos libre. Esta opción, tras un proceso previo de búsqueda bibliográfica, identificación y selección de candidatas, contacto con desarrolladores... se basó, según Moreno y Cerverón (2006, p. 2), especialmente "en su adaptación al modelo de docencia de asignatura con grupos y subgrupos, la posibilidad de usar comunidades de investigación o de gestión y en la oportunidad de aprovechamiento mutuo de la experiencia de la UNED con .LRN". La implantación se ha desarrollado en varias fases desde el período de análisis en octubre de 2003 encontrándose en el presente curso académico, aunque el uso es voluntario, con todos los cursos y usuarios activados: unos 50.000 estudiantes, 3.500 profesores, 6.300 cursos y 23 comunidades.

Esta aplicación digital permite, entre otras opciones, la publicación de materiales docentes, enviar noticias a los grupos docentes, planificar las sesiones de trabajo, también incluye el correo de los miembros del grupo y la gestión de entrega y corrección de los trabajos de los alumnos, forum y chats, y también puede utilizarse por grupos de investigación. Y, después del período de implantación, se plantean tareas tanto técnicas como pedagógicas de ajuste y formación dentro de la universidad así como continuar las líneas de colaboración establecidas.

También se le denomina Aula Virtual al entorno virtual de enseñanza y aprendizaje de la Universitat Jaume I. Sin embargo, en este caso la plataforma elegida ha sido Moodle, tras el pertinente proceso de evaluación de algunos de los sistemas creados con SL. Esta plataforma también ha sido adoptada por varias universidades españolas: Las Palmas de Gran Canaria, Málaga, Politècnica de Catalunya, y extranjeras tan prestigiosas como la Open University. Cuenta, además, con una importante comunidad de desarrolladores de la que forma parte la Universitat Jaume I.

En esta universidad se optó por este entorno, según reza en el informe realizado (2004, p. 14) ${ }^{11}$ por "su combinación de flexibilidad y sofisticación didáctica, por su flexibilidad tecnológica, por el dinamismo de su comunidad de desarrollo y por su facilidad de uso para estudiantes y profesores". En la actualidad está integrado en la base de datos de gestión académica de la UJI y entre las actividades que se pueden 
realizar destacan los accesos a foros, diálogos, consultas electrónicas, evaluar trabajos, distribuir materiales de aprendizaje, mensajería instantánea... asimismo se puede usar por grupos de investigación que requieren de un espacio virtual para su trabajo.

Cerramos esta breve revisión señalando algunas coincidencias y también desencuentros. En primer lugar, que estas dos últimas universidades han abierto procesos de evaluación de plataformas de apoyo a la docencia basadas en SL, como así consta en los informes o comunicaciones presentadas. En segundo lugar, que en la muestra final ambas han preseleccionado básicamente a las mismas candidatas (Atutor, Moodle y .LRN en la Universitat Jaume I, Atutor, Moodle, ILIAS y .LRN en la Universitat de València). Sin embargo observamos que la elegida ha sido diferente en ambos procesos. ¿A qué responde este desencuentro en la opción tecnológica final? ¿No habría sido más razonable y a la vez económico aunar esfuerzos para el desarrollo de la misma plataforma?

\section{Potencial innovador del SL}

Hay un consenso bastante generalizado en torno a que el SL es resultado y estímulo de la innovación en el ámbito de las tecnologías informáticas (Castells, 2006, p. 264). Lo cual se constata no sólo en su rápida mejora técnica, sino también en cuanto al tejido social con el que se rodea y sin el cual no sería comprensible. Ahora bien, la cuestión es si el potencial innovador que caracteriza al SL se proyectará también a las actividades en las que se aplican tales herramientas, las enseñanzas universitarias en nuestro caso. La controversia surge de inmediato y no tanto por las posibilidades "didácticas" de este software, como por la dificultad añadida a la que han de hacer frente los usuarios, principalmente el profesorado que puede ser algo más remiso a la utilización de las tecnologías en sus clases. Cuando aún no ha "aprendido" a manejar el software propietario, resulta que ahora debe migrar hacia el libre que, además, se suele decir, es menos amigable que el propietario.

No conocemos trabajos de campo que analicen esta cuestión concreta, a nuestro entender fundamental para la pedagogía de las tecnologías informáticas en la enseñanza. De momento los estudios disponibles no van mucho más allá de resaltar las aportaciones del SL al ámbito concreto de la docencia universitaria. Desde esta perspectiva aparecen voces que apuestan por su oportunidad e idoneidad en aras de mejorar el proceso de enseñanza-aprendizaje en las aulas universitarias, dirección en la que apuntan autores como Bulchand (2005) o Bustamante (2005), entre otros. 
Las líneas argumentales de estos trabajos coinciden en señalar mayormente que la filosofía subyacente al SL es convergente con la de la Universidad, ya que pretende compartir el conocimiento, fomentar la colaboración frente a la competitividad, democratizar el acceso a la información, etc. E incluso van más allá, proponiendo el SL como un elemento posibilitador de la transformación de la Universidad, desde un modelo jerárquico a un modelo de organización más flexible, un modelo en red que se adapte más fácilmente a la Sociedad del Conocimiento. Quizá sea preciso insistir en que esta posibilidad se hará realidad dependiendo no tanto de la tecnología en sí, no del SL, sino del cambio en las actitudes, en las creencias y en las prácticas docentes universitarias. Como afirma Area (2004, p. 222) "el reto de futuro está en que las universidades innoven no sólo su tecnología, sino también sus concepciones y prácticas pedagógicas, lo que significa modificar el modelo de enseñanza universitario en su globalidad...”.

\section{CONCLUSIONES}

Aunque aún no se disponen de demasiados datos sobre el fenómeno, sí hay ya bastante literatura que permite caracterizar la estructura que está adoptando en estos momentos el espacio controversial que nos ocupa. Sobre la base de la referencia a las tres universidades valencianas citadas más arriba se pueden extraer, con carácter provisional, algunas consideraciones finales.

- Los discursos y las prácticas que rodean al SL lo convierten en el agente capaz de cumplir la función de refocalización del espacio controversial definido en torno a la aplicación pedagógica de las tecnologías informáticas. Desde luego que las universidades, en general, ejercen un importante papel de liderazgo tanto en el plano de desarrollo como de difusión del software no propietario. Pese a lo cual, la posición institucional es ambivalente al hacer cohabitar a los dos tipos de tecnología.

- Quizá por este papel de socialización que están cumpliendo las universidades, las administraciones educativas convenien con ellas la formación del profesorado de las etapas no universitarias en el manejo del SL. Si bien los contratos mejor dotados y orientados al desarrollo de aplicaciones, al menos en la Comunidad Valenciana, se los lleven equipos y empresas ajenos a la universidad. De modo que resulta revelador, y en ello continuaremos trabajando, preguntarse por el tamiz desde el que las universidades interpretan la "libertad" y la "gratuidad" asociadas al SL. 
- Las controversias en torno al SL se mueven todavía en el ámbito de lo técnico. Las innovaciones se revisan y reformulan en los abundantes foros (congresos, jornadas, grupos de trabajo) y redes en las que los responsables informáticos de las tres universidades participan. Sin embargo, estas controversias no han llegado al ámbito de lo pedagógico, paradójicamente cuando son las plataformas de e-learning las que operan con SL y no tanto las aplicaciones de gestión de las universidades.

- Uno de los aspectos más innovadores del SL son sus estrategias para aprovechar las sinergias y socializar los hallazgos, ambas características de difícil acomodo en el plano pedagógico que aún impera en las aulas universitarias. Se puede compartir la herramienta informática, caso del e-learning, pero sin que ello alcance plenamente al plano didáctico que sigue siendo jurisdicción de cada docente. Quizá porque el SL no es tan libre como se dice ni ha logrado desprenderse del marchamo "técnico" que rodea a las tecnologías informáticas, como tampoco las pesadas estructuras universitarias son tan permeables a las políticas de libertad que se proclama de una determinada tecnología.

\section{NOTAS}

1 Desarrollado en la Tesis Doctoral de José Peirats Chacón: "Variantes organizativas generadas por las Tecnologías de la Información. Un estudio en los centros de primaria de la Comunidad Valenciana" (Universitat de València, 2006).

2 Disponible en: http://www.mec.es/mecd/gabipren/documentos/ProyectoLOU.pdf [consulta 2007, 10 de abril].

3 Disponible en: http://www.mec.es/universidades/eees/files/Documento Marco.pdf [consulta 2007, 1 de abril].

4 Informe Anual sobre el desarrollo de la Sociedad de la Información en España. Elaborado por la Fundación France Telecom España. Disponible en: http://www. fundacionauna.com/areas/25 publicaciones/eEspaña 2006.pdf [consulta 2007, 10 de marzo].

5 El País [consulta 2007, 4 de febrero].

6 Motorola, NEC, NTT DoCoMo, Panasonic Mobile Communications, Samsung Electronics y Vodafone. Levante EMV [consulta 2007, 31 de enero].

7 En http://es.wikipedia.org

8 Responsable de desarrollo de mercado Linux y Open Source en IBM. Ciberp@ís [consulta 2007, 25 de enero].

9 Según se puede leer en: http://barrapunto.com/articles/04/07/21/1328256.shtml [consulta 2007, 3 de abril].

10 Entre otras la Universidad de Viena, Heidelberg o Bergen en Europa, MIT y Harvard en UA o la Universidad de Galileo en Guatemala.

11 Se puede consultar el informe realizado en el curso 2003-2004, donde se detalla los criterios y la metodología empleados en el proceso de evaluación así como las 
conclusiones alcanzadas. Disponible en: http://cent.uji.es/doc/eveauji es.pdf [consulta 2007, 10 de marzo].

12 Definición que podemos leer en: http://www.fsf.org/licensing/essays/free-sw.html [consulta 2007, 10 de abril].

13 Para conocer algo más de ellas es interesante el artículo de De las Heras, Q. y González, J. (2001). Actualidad del Software Libre. Novática, 154, pág. 7 y ss.

14 Ver por ejemplo De las Heras y González (2001), o las pág. 49 y ss. del Dossier de la III Conferencia Internacional de Software Libre, celebrada en Badajoz del 7 al 9 de Febrero de 2007. Disponible en: http://www.freesoftwareworldconference.com/ files/dossier es.pdf [consulta 2007, 1 de abril].

15 Según se lee en: http://www.softwarelibre.ulpgc.es/ [consulta 2007, 5 de marzo].

16 En: http://usuarios.lycos.es/nanoelduende/linux/propuesta.html [consulta 2007, 8 de abril].

17 http://www.uji.es/bin/uji/norm/estatuts/estf-cas.pdf [consulta 2007, 2 de febrero].

18 Distribución GNU/Linux creada por la Conselleria de Cultura, Educació i Esports y orientada al sistema educativo valenciano. Lliurex está basado completamente en software libre y gratuito, y fue presentado en un espectacular congreso realizado a finales del curso 2003/2004 en la ciudad de Valencia.

19 En: http://lliurex.net/moodle [consulta 2007, 10 de febrero].

\section{REFERENCIAS BIBLIOGRÁFICAS}

Area Moreira,M.(2000).¿QuéaportaInternet al cambio pedagógico en la Educación Superior?, en: Pérez, R. (Coord): Redes multimedia y diseños virtuales. En las Actas del III Congreso Internacional de Comunicación, Tecnología y Educación. Universidad de Oviedo, 128-135. [en línea] Disponible en: http://webpages. ull.es/users/manarea/Documentos/ documento7.htm [consulta 2007, 10 de febrero]

Area Moreira, M. (2004). Los medios y las tecnologías en la educación. Madrid: Pirámide.

Bulchand, J. (2005). Software libre en la Universidad, en: II Libro Blanco del Software Libre en España. [en línea] Disponible en: http://www.libroblanco. com/document/II libroblanco del software libre.pdf [consulta 2007, 1 de febrero]

Bustamante, J. (2005). Software libre y Universidad, en: II Libro Blanco del Software Libre en España. [en línea]
Disponible en: http://www.libroblanco. com/document/II libroblanco del software libre.pdf [consulta 2007, 1 de febrero]

Cabero, J. (Dir.) (2002). Las TICs en la Universidad. Sevilla: MAD.

Carnoy, M. (2004). Las TIC en la enseñanza: posibilidades y retos. Lección inaugural del curso académico 2004-05 de la UOC. [en línea] Disponible en: http:// www.uoc.edu/inauguralo4/dt/esp/ carnoy1004.pdf [consulta 2006, 6 de febrero]

Castells, M. (2006). Observatorio global. Crónicas de principios de siglo. Barcelona: La Vanguardia Ediciones.

Comisión Europea (2000). Free Software/ Open Source: Information Society Opportunities for Europe? [en línea]. Disponible en: http://eu.conecta.it/ paper.pdf [consulta 2007, 1 de enero]

Conferencia de Rectores de Universidades Españolas (2006). Las TIC en el sistema universitario español. [en 
línea] Disponible en: http://www. crue.org/UNIVERSITIC2006/ Analisis\%20Estrategico.pdf [consulta 2007, 20 de abril]

De las Heras, Q.; González, J. (2001). Actualidad del Software Libre. Novática, 154, 5-12.

De Pablos, J.; Villaciervos, P. (2005). El espacio europeo de educación superior y las tecnologías de la información y la comunicación. Percepciones y demandas del profesorado. Revista de Educación, 337, 99-124.

Fernández, R. (2005). El software libre o la paradoja del altruismo. Novática, 178, 66-68.

Figuerola, C. (2007). El uso de software libre en los sitios web universitarios españoles. En las Actas del FLOSS International Conference, celebrado en Jerez. [en línea] Disponible en: http://softwarelibre.uca. es/jornadas/fic/spa/materiales/actas. pdf [consulta 2007, 1 de marzo]

Fundación Auna (2005). Informe anual sobre el desarrollo de la Sociedad de la Información en España, [en línea]. Disponible en: http://www. fundacionauna.com/areas/25 publicaciones/EESPA A2005 COMPLETO V3.pdf [consulta 2007, 1 de enero]

González, T. (2005). El espacio europeo de Educación Superior: Una nueva oportunidad para la Universidad, en: Colás, P.; De Pablos, J. (Coords): La Universidad en la Unión Europea: El espacio europeo de Educación superior y su impacto en la docencia. Málaga: Aljibe, (27-55).

Gore, J. M. (1996). Controversias entre las pedagogías. Madrid: Morata.

Himanen, P. (2002). La ética del hacker y el espíritu de la era de la información. Madrid: Destino.

Marqués, P. (2000). Impacto de las TIC en la enseñanza universitaria [en línea]. Disponible en: http://dewey.uab.es/ pmarques/ticuniv.htm [consulta 2007, 11 de enero]

Marrero, J. (2004). Sociedad de la información y dinámica mediadora de la universidad. Qurriculum: revista de teoría, investigación y práctica educativa, 17, 17-46.

Martín Gordillo, M. (Coord.) (2006). Controversias tecnocientíficas. Diez casos simulados sobre ciencia, tecnología, sociedad y valores. Barcelona: Octaedro-OEI.

Martín, M. A.; Aguiar, M. V. (2004). Protocolo de introducción de software libre a personal docente e investigador universitario y diseño de un proyecto formativo [en línea]. Relatec, 1 (3), 511528. Disponible en: http://www.unex. es/didactica/RELATEC/sumario 3 1.htm [consulta 2007, 12 de enero]

Martínez, F. (Dir.) (2002). Aplicación de las nuevas tecnologías a la actividad del profesorado universitario. Huelva: Universidad de Huelva.

Moreno, P.; Cerverón, V. (2006). Plataforma tecnológica para potenciar los procesos de enseñanza-aprendizaje: desarrollo en la Universitat de València basado en software libre y colaborativo. Ponencia presentada en el VIII Simposio Internacional de Informática Educativa SIIE2006. [en línea] Disponible en: http://aulavirtual.uv.es [consulta 2007, 8 de enero]

Nudler, O. (2004). Hacia un modelo de cambio conceptual: espacios controversiales y refocalización. Revista de Filosofía, 29 (2), 7-19.

Romeo, A. y García, J. (2003). La pastilla roja. Software libre y Revolución digital. Madrid: Edit Lin.

Valverde, J. (2004). Educación superior y entornos virtuales de aprendizaje: evolución dela oferta formativa on-line en las universidades públicas. Qurriculum: revista de teoría, investigación y práctica educativa, 17, 95-118. 


\section{PERFIL ACADÉMICO Y PROFESIONAL DE LOS AUTORES}

José Peirats Chacón. Licenciado en Ciencias de la Educación por la Universidad de Valencia. Profesor asociado de la Universitat de València y Doctor en Pedagogía. Líneas de investigación relacionadas con la Educación Especial y con las TIC en las organizaciones educativas de las que ha presentado varias publicaciones, entre ellas: Educación Especial: Orientaciones prácticas (Aljibe, 2005). Políticas institucionales y trabajo colaborativo entre docentes: El ejemplo de la Zona Clic. (Revista Iberoamericana de Educación, 2005).

E-mail: jose.peirats@uv.es

Ángel San Martín Alonso. Profesor titular de Didáctica y Organización Escolar en la Universitat de València, miembro del grupo de investigación CRIE, se viene ocupando del estudio de las sinergias culturales y organizativas entre las TIC y los centros escolares. Ha publicado libros como La escuela de las tecnologías o Del texto a la imagen y contribuciones a libros como Para una tecnología educativa o Las nuevas tecnologías en la enseñanza, además de numerosos artículos como La digitalización de la enseñanza o La competencia desleal del e-learning con los sistemas escolares nacionales.

\section{E-mail: angel.sanmartin@uv.es}

Cristina Sales Arasa. Doctora en Pedagogía por la Universitat de València. Psicopedagoga en la Enseñanza Secundaria. Profesora Asociada en la Universitat de València (2003-2004). Líneas de investigación relacionadas con las TIC en el ámbito educativo, que se plasman en publicaciones como Las tecnologías de la información en las estrategias metodológicas del profesorado de secundaria: ¿Qué criterios orientan sus decisiones? (Actas del II Congreso online del Observatorio para la Cibersociedad, 2004) o Las TI y la actividad escolar (Educación y Medios, 2000).

E-mail: cristina.sales@uv.es

DIRECCIÓN DE LOS AUTORES

Departament de Didàctica i Organització

Escolar

Universitat de València

C/ Blasco Ibáñez, nº 30

46010 Valencia

Fechas de recepción del artículo: 17/10/07

Fechas aceptación del artículo: 09/01/08 